\title{
Collapsing Schwarzschild Interior Solution
}

\author{
Rainer Burghardt \\ A-2061 Obritz 246, Austria \\ Email: arg@aon.at
}

Received 12 September 2015; accepted 25 October 2015; published 29 October 2015

Copyright (C) 2015 by author and Scientific Research Publishing Inc.

This work is licensed under the Creative Commons Attribution International License (CC BY). http://creativecommons.org/licenses/by/4.0/

(c) (i) Open Access

\begin{abstract}
We extend the static interior Schwarzschild solution to a collapsing model by applying geometrical methods. We examine the field quantities and field equations in the comoving and non-comoving observer systems. The collapsing stellar object contracts asymptotically to its minimum extent and needs an infinitely long time to arrive at the final state. The event horizon of the exterior Schwarzschild solution is not reached or even crossed. A geometric model of ECOs (eternally collapsing objects) is presented.
\end{abstract}

\section{Keywords}

Collapse, Interior Schwarzschild Solution, Interior Horizon, Comoving and Non-Comoving Reference Systems

\section{Introduction}

Since Oppenheimer and Snyder [1], inspired by an expanding cosmological model of Tolman, in 1939 first proposed a model for a collapsing star, many authors have adopted this problem. Among the many suggestions are only a few exact solutions of Einstein's field equations. The reason is that the Einstein field equations are underdetermining and although the conservation laws have been consulted there are not enough equations available to determine the metric coefficients and the physical quantities of the matter configuration.

Therefore we do not try to solve the Einstein field equations, but we have numerous conjectures and make some assumptions. Then we try to assemble them all hoping to obtain a suitable model for the collapse of a star:

1) We rely on geometric ideas which have proven for the exterior and interior Schwarzschild solutions. We interpret the space-like part of the interior solution as the cap of a sphere and the space-like part of the exterior solution as Flamm's paraboloid.

2) We consider the time-like part of the metric to be an element of a double-surface. This is what we have discussed in [2] in detail, but here we will indicate this only briefly.

3) The two solutions, the interior and the exterior, are embeddable into a 5-dimensional flat space, but six variables are required, two of them lie in one and the same dimension. Thus, the theorems of Kasner and 
Eisenhart are not violated.

4) The methods of embedding provide strong support for the design of the model. The fact that this is not an academic exercise has been confirmed by us in the discovery of new interior solutions for the models by Kerr, Kerr-Newman, NUT, and Reissner-Nordström [2].

5) The complete geometry consists of a spherical cap which slides down on Flamm's paraboloid during the collapse. The exterior Schwarzschild geometry remains unchanged according to Birkhoff's theorem. The collapse causes no change in the exterior gravitational field. Above all it produces no gravitational waves.

6) We perform all calculations by using the method of tetrads. It will prove to be very advantageous for constructing a collapsing model. The Ricci-rotation coefficients guarantee that we will have direct access to the physical and geometrical quantities.

7) The question of the linking condition on the boundary surface of the interior and exterior solutions should be clarified. Israel [3], O’Brien and Synge [4], Robson [5], Bonnor and Vickers [6], Nariai [7], and Lichnerowicz [8] extensively have written on this subject. We want to emphasize that we have to approach to this problem carefully. One has to match a time-dependent model to a static one. At any given moment $t=$ const. the metric of the interior solution at the boundary should match the exterior one, i.e., the two geometries should be connected. Furthermore, the first derivatives of the metric should match, i.e. the two geometries should have common tangents at the boundary surface. In the present case this can only mean that the spherical cap and Flamm's paraboloid have a common cutting tangent at the boundary surface. Expressions which do not describe the basic geometry, but the change of geometry, i.e. the collapse, cannot be taken into consideration with respect to the linking, by any means. That these expressions can be problematic shows us a look at historical studies. Although the metrics of the model of Oppenheimer and Snyder match the derivatives of the metrics do not match. Nariai and Tomita [9] [10] are of opinion that the interior OS solution does not match the exterior Schwarzschild solution and they replace the exterior Schwarzschild solution by a more complicated one while they maintain the interior OS solution. They have attempted to incorporate into the matching also the dynamic quantities which have their origin in the collapse. This presupposes that there is a surface which supports the dynamic properties of the model. We believe that the theory of surfaces does not provide in general such properties.

8) Accordingly, there is no "collapsing metric", i.e. there is no line whose element can be written down. There is in general no surface $\Sigma_{c}$ that geometrically describes the collapse, i.e. a graphic surface or an abstract Riemannian manifold on which one could draw such a line. A look at Figure 1 shows that the model can be completely described by the inner surface and the outer surface of the whole Schwarzschild model. There is no global coordinate system that could cover such a surface $\Sigma_{\text {c }}$. The physical and geometrical quantities are presented in a reference system by using tetrads. We introduce a reference system which is linked to an observer who comoves with the collapse and another reference system which does not comove with the collapse. Both systems are connected by a Lorentz transformation with non-constant velocity parameters.

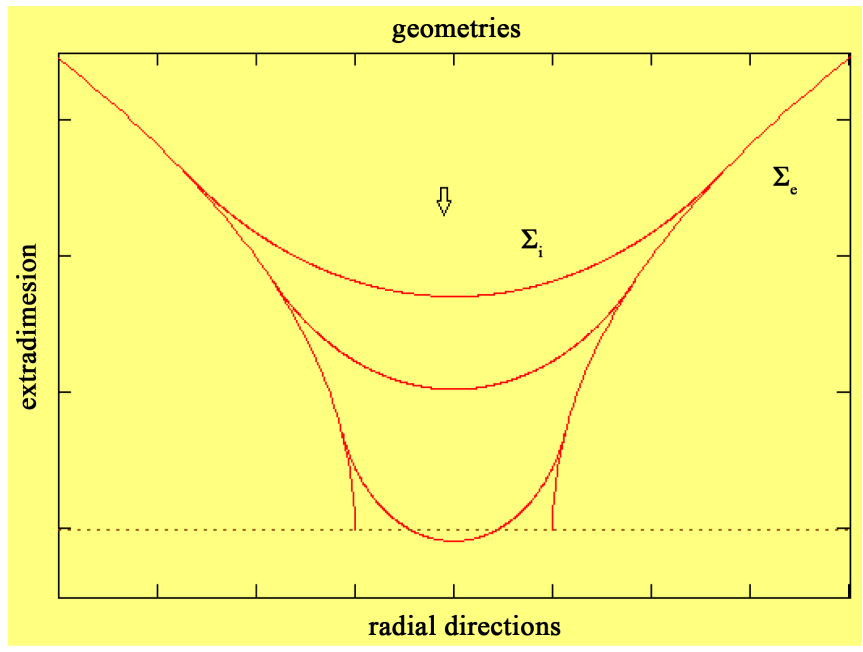

Figure 1. The inner surface and the outer surface of the whole Schwarzschild model. 
9) For the reasons mentioned above, the allocation of a common coordinate system, or the transition from the comoving to the non-comoving coordinate system respectively, is problematic. Oppenheimer and Snyder were there partly successful. McVittie [11] [12] gets stuck half way. For the transformation of the time, Weinberg [13] writes down an integral whose solution, however, he does not specify. Many authors do not lay a hand on this problem.

10)The collapsing solution is based on the static interior Schwarzschild solution taking into account the pressure inside the matter. The pressure and the mass density are time-dependent. The stellar object cannot be interpreted as an incompressible homogeneous fluid sphere any longer.

11)Pressure and density of matter should never be infinitely high. The stellar object should not shrink to a point and the curvature of space cannot be infinitely high. As final state no singularity should emerge.

12)The static model has a horizon. At a relatively small radius of the object the pressure at its center would be infinite. Further, after drilling a hole through the center of the star we make a body oscillate through it. If the object took a certain minimal radius, the body would reach the velocity of light falling through the center of the star [14]. We demand that this inner horizon specifies the minimum extension of the collapsing star.

All of that we put together into a toolbox, from which we take out elements as required.

\section{The Interior Schwarzschild Solution}

Since we intend to create a collapsing model based on the static interior Schwarzschild solution, we are arranging it formally in such a way that an extension is possible. We work out essential parts for the collapse in greater detail and we focus on the geometrical background of the model. This paves the way for us to modify the model so that it can describe a collapse.

The interested reader is supported with numerous detailed calculations in [15]. A more detailed description of the interior solution can be found in [2]. The interior Schwarzschild solution is based on the seed metric

$$
\mathrm{d} s^{2}=\alpha_{R}^{2} \mathrm{~d} r^{2}+r^{2} \mathrm{~d} \vartheta^{2}+r^{2} \sin ^{2} \vartheta \mathrm{d} \varphi^{2}-a_{R}^{2} \mathrm{~d} t^{2}, \quad a_{R}=\sqrt{1-r^{2} / R_{g}^{2}}, \quad \alpha_{R}=1 / a_{R} .
$$

Herein $R_{g}=$ const. is the radius of the spherical cap which is used to describe the spatial part of the model and

$$
r=R_{g} \sin \eta
$$

the radial variable in the 5-dimensional flat embedding space, $\eta$ the polar angle which is simultaneously the angle of ascent of the spherical cap.

Of the quantity

$$
v_{R}=-\frac{r}{R_{g}}=-\sin \eta
$$

we frequently make use later on. From $a_{R}^{2}+v_{R}^{2}=1$ immediately results $a_{R}=\cos \eta$. Thus, the seed metric takes the form

$$
\mathrm{d} s^{2}=\frac{1}{\cos ^{2} \eta} \mathrm{d} r^{2}+r^{2} \mathrm{~d} \vartheta^{2}+r^{2} \sin ^{2} \vartheta \mathrm{d} \varphi^{2}-\cos ^{2} \eta \mathrm{d} t^{2}
$$

and formally corresponds to the de Sitter metric. However, it does not describe a spherical space, but a spherical cap. The connection between the tetrad differentials and the coordinate differentials is established with $\mathrm{d} x^{m}=\stackrel{m}{e}_{i} \mathrm{~d} x^{i}$, whereby is $\mathrm{d} x^{i}=\{\mathrm{d} r, \mathrm{~d} \vartheta, \mathrm{d} \varphi, i \mathrm{~d} t\}$. From (2.1) we read the 4-bein system

$$
\stackrel{1}{e_{1}}=\alpha_{R}, \quad \stackrel{2}{e}=r, \quad \stackrel{3}{e}=r \sin \vartheta, \quad \stackrel{4}{e_{4}}=a_{R}
$$

and we calculate the Ricci-rotation coefficients

$$
A_{m n}^{s}=e_{i_{[n \mid m]}}^{s} e^{i}+g^{s r} g_{m t} e_{i[n \mid r]}^{t} e^{i}+g^{s r} g_{n t} e_{i_{[m \mid r]}^{t}}^{e^{i}} .
$$

The indices $(m, n, \cdots)$ number the 4-bein, the (i) are coordinates indices. We prefer to present the variables of

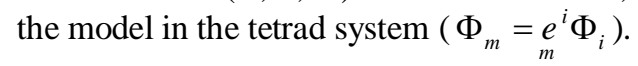


We decompose the Ricci-rotation coefficients into

$$
A_{m n}^{s}=B_{m n}^{s}+C_{m n}^{s}+\hat{U}_{m n}^{s} .
$$

With the following unit vectors

$$
b_{m}=\{0,1,0,0\}, \quad c_{m}=\{0,0,1,0\}, \quad u_{m}=\{0,0,0,1\}
$$

we write

$$
B_{m n}^{s}=b_{m} B_{n} b^{s}-b_{m} b_{n} B^{s}, \quad C_{m n}^{s}=c_{m} C_{n} c^{s}-c_{m} c_{n} C^{s}, \quad \hat{U}_{m n}^{n_{s}^{s}}=u_{m} U_{n} u^{s}-u_{m} u_{n} U^{s} .
$$

We calculate the two lateral field quantities $B$ and $C$ and the acceleration $\hat{U}$ from (2.6) with

$$
B_{1}=-e_{2}^{2} e_{2 \mid 1}^{2}, \quad C_{1}=-e_{3}^{3} e_{3 \mid 1}^{3}, \quad C_{2}=-e_{3}^{3} e_{3 \mid 2}^{3}, \quad \hat{U}_{1}=-e_{4}^{4} e_{4 \mid 1}^{4}
$$

using

$$
\partial_{1}=a_{R} \frac{\partial}{\partial r}, \quad \partial_{2}=\frac{1}{r} \frac{\partial}{\partial \vartheta}, \quad \partial_{3}=\frac{1}{r \sin \vartheta} \frac{\partial}{\partial \varphi}, \quad \partial_{4}=\frac{1}{a_{R}} \frac{\partial}{i \partial t} .
$$

Finally we obtain [15 \#1 - \#3]

$$
B_{m}=\left\{\frac{a_{R}}{r}, 0,0,0\right\}, \quad C_{m}=\left\{\frac{a_{R}}{r}, \frac{1}{r} \cot \vartheta, 0,0\right\}, \quad \hat{U}_{m}=\left\{\alpha_{R} v_{R} \frac{1}{R_{g}}, 0,0,0\right\} .
$$

Thus, we have derived the field quantities of the seed metric and also explained the notation.

We realize that the seed metric is too simple. The acceleration is directed outwards and does not match the corresponding exterior Schwarzschild value. Nevertheless, we calculate the Ricci and the curvature scalar, because the gained structures are retained in the transition to both the genuine interior Schwarzschild model and the collapsing model as well. From

$$
R_{m n}=A_{m n \mid s}^{s}-A_{n \mid m}-A_{r m}{ }^{s} A_{s n}^{r}+A_{m n}{ }^{s} A_{s}, \quad A_{n}=A_{r n}^{r}
$$

and (2.7), (2.9) we obtain with [15 \#7]

$$
\begin{aligned}
& R_{m n}=-\left[\hat{U}^{\widehat{s}}{ }_{1 \mid s}^{s}+U^{s} U_{s}\right] h_{m n} \\
& -\left[B_{n \| m}+B_{n} B_{m}\right]-b_{n} b_{m}\left[B_{\| s}^{s}+B^{s} B_{s}\right] \\
& -\left[C_{n \| m}+C_{n} C_{m}\right]-C_{n} C_{m}\left[C_{\| s}^{s}+C^{s} C_{s}\right]
\end{aligned}
$$

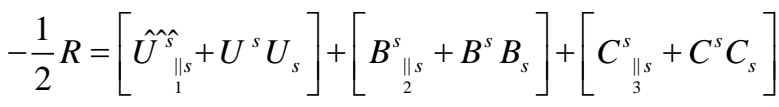

where by the graded derivatives [2]

$$
\hat{U}_{\substack{n \| m \\ 1}}^{m m}=U_{n \mid m}, \quad B_{n \| m}=B_{n \mid m}-U_{m n}^{s} B_{s}, \quad C_{\substack{n \| m \\ 3}}=C_{n \mid m}-U_{m n}^{s} C_{s}-B_{m n}^{s} C_{s}
$$

prove to be highly advantageous and are almost mandatory for understanding the structures of the model. Therein is

$$
h_{m n}=\operatorname{diag}(1,0,0,1)
$$

a submatrix of the tetrad metric $g_{m n}=\operatorname{diag}(1,1,1,1)$. For later processing we note the subequation of the Ricci [15 \# 6]

$$
\hat{U}_{\substack{\uparrow \\{ }_{1}}}^{\widehat{s}}+U^{s} U_{s}=-\frac{1}{R_{g}^{2}}
$$


The entire Einstein tensor is calculated by using [15 \# 1 - \# 5].

We manage the transition to the genuine interior Schwarzschild metric with the projector $P_{m}^{s}$ which has only the few components

$$
P_{1}^{1}=P_{2}^{2}=P_{3}^{3}=1, \quad P_{4}^{4}=P, \quad P=-\frac{1}{2} \frac{a_{R}}{a_{T}}, \quad a_{T}=\frac{1}{2}\left(3 a_{R}^{g}-a_{R}\right)
$$

different from 0 . Therein is $a_{R}^{g}$ the value of $a_{R}$ on the surface of the star. If one matches the cap of the sphere to Flamm's paraboloid the relation

$$
\rho_{g}=2 R_{g}, \quad \rho=\sqrt{\frac{2 r^{3}}{M}}
$$

has to apply.

$\rho$ is the radius of curvature of the Schwarzschild parabola, $\rho_{g}$ its value at the surface of the star. Thus, with

$$
R_{g}=\sqrt{\frac{r_{g}^{3}}{2 M}}
$$

one has made accessible the relation between the radius of the spherical cap and the position $r_{g}$ of the star's surface in the embedding space. The geometric relations (2.17) are shown graphically in [2]. Obviously, the projector (2.16) operates effectively only on the fourth components of the quantities. If we from now on consistently use hats for the quantities of the seed metric, we have

$$
\mathrm{d} x^{m}=\left(P^{-1}\right)_{s}^{m} \mathrm{~d} \hat{x}^{s}, \quad \partial_{m}=P_{m}^{s} \hat{\partial}_{s}, \quad A_{m n}{ }^{r}=P_{m}^{s} A_{s n}{ }^{r} .
$$

To understand the first operation in (2.19) we replace the time differential in the line element (2.1) by

$$
i d \hat{t}=-R_{g} d i \psi \text {. }
$$

We interpret the time interval as arc element on a pseudo-circle and we let operate the projector on it

$$
\mathrm{d} x^{4}=\left(P^{-1}\right)_{4}^{4} \mathrm{~d} \hat{x}^{4}=-2 \frac{a_{T}}{a_{R}}\left(-a_{R} R_{g} \mathrm{~d} i \psi\right)=a_{T} 2 R_{g} \mathrm{~d} i \psi, \quad i \mathrm{~d} t=2 R_{g} \mathrm{~d} i \psi, \quad \mathrm{d} x^{4}=a_{T} i \mathrm{~d} t
$$

and we get the interior Schwarzschild line element

$$
\mathrm{d} s^{2}=\alpha_{R}^{2} \mathrm{~d} r^{2}+r^{2} \mathrm{~d} \vartheta^{2}+r^{2} \sin ^{2} \vartheta \mathrm{d} \varphi^{2}-a_{T}^{2} \mathrm{~d} t^{2}
$$

If one takes into account (2.18) and $a_{T}^{g}=a_{R}^{g}=\sqrt{1-2 M / r_{g}}$, then this line element related to the surface of the star coincides with the one of the exterior field. If we write the time-like part as

$$
\mathrm{d} x^{4}=a_{T} 2 R_{g} \mathrm{~d} i \psi=\left(3 R_{g} \cos \eta_{g}-R_{g} \cos \eta\right) \mathrm{d} i \psi
$$

we realize that the time which we read from our clock is the real accompanying number of a rotation process through an imaginary angle $i \psi$.

The projector technology gets its actual entitlement only within a 5-dimensional embedding theory [2]. However, here we have shown it in outline, because it plays a useful role in the remodeling of the static model to a collapsing model.

The third operation (2.19) affects only the quantity $\hat{U}$

$$
U_{m n}{ }^{s}=P_{m}^{r} \hat{U}_{r n}^{s}, \quad U_{1}=P U_{1} .
$$

A simple calculation [15 \# 9] shows that now holds

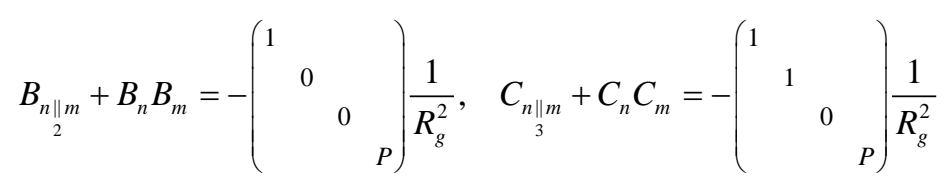

because now the quantity $U_{m n}^{s}$ containing $P$ occurs in the graded derivative. With [15 \# 8] one calculates for $U^{s}$ 


$$
U_{1}^{s}{ }_{1}^{s}+U^{s} U_{s}=-\frac{P}{R_{g}^{2}}
$$

The field quantity $E_{1}$ in

$$
U_{1}=A_{41}^{4}=-e_{4}^{4} e_{4 \mid 1}^{4}=\frac{1}{a_{T}} a_{T \mid 1}=-E_{1}, \quad E_{1}=-P \hat{U}_{1}=\frac{v_{R}}{a_{T}} \frac{1}{\rho_{g}}
$$

is the gravitational force inside the star, is directed inwards, and matches the Schwarzschild term $E_{1}=\alpha_{R} v_{R} \frac{1}{\rho}$ on the surface [2].

If one removes the hats from (2.12), one has the structure of the Einstein tensor of the Schwarzschild model and with

$$
T_{m n}=-p g_{m n}+\left(p+\mu_{0}\right) u_{m} u_{n}
$$

gains the expressions for the matter configuration

$$
\kappa p=-\frac{1}{R_{g}^{2}}(1+2 P), \quad \kappa \mu_{0}=\frac{3}{R_{g}^{2}}, \quad 1-P=\frac{3}{2} \frac{a_{T}^{g}}{a_{T}}, \quad p+\mu_{0}=(1-P) \frac{2}{R_{g}^{2}}=\frac{a_{T}^{g}}{a_{T}} \mu_{0}
$$

and finally the equation of state

$$
p=\left(\frac{a_{T}^{g}}{a_{T}}-1\right) \mu_{0} .
$$

The relations (2.26) are applied to the collapsing model. The pressure can also be written as

$$
\kappa p=-\frac{3}{R_{g}^{2}} \frac{\cos \eta_{g}-\cos \eta}{3 \cos \eta_{g}-\cos \eta}
$$

From (2.2) one obtains $\eta=0$ for $r=0$. Thus, in the center of the star $(\eta=0)$ is

$$
\kappa p_{c}=\frac{3}{R_{g}^{2}} \frac{1-\cos \eta_{g}}{3 \cos \eta_{g}-1} \text {. }
$$

For $\cos \eta_{g}=1 / 3$ the pressure in the center of the star is infinite. If one makes use of this in (2.3) and (2.18), one can see that a star cannot be arbitrarily small. In dependence on its mass its radial coordinate in the embedding space must be

$$
r_{h}>\frac{9}{4} M .
$$

This is of considerable importance for the collapse. We expect that a star can shrink only close to this minimum value. This is particularly interesting because $r_{h}$ is above the event horizon of the exterior solution.

For later use, we calculate from (2.16)

$$
\frac{1}{P} P_{\mid 1}=\frac{1}{a_{R}} a_{R \mid 1}-\frac{1}{a_{T}} a_{T \mid 1}=\hat{U}_{1}-U_{1}
$$

and with (2.22)

$$
P_{\mid 1}=(1-P) U_{1}
$$

Thus, we have worked out all the basic relations and we have brought the Schwarzschild model into a form which allows us to extend the interior Schwarzschild solution in such a way that a collapse of a simple matter configuration can be described.

\section{Collapse, Comoving Observer System}

After the arrangements in the last section, we are able to reflect, how the inner part of the complete Schwarzschild 
solution can be related to a collapse. We let the spherical cap, which represents geometrically the region of the interior solution, slide down the Schwarzschild parabola.

Evidently, thereby the radius of the spherical cap changes. Thus, $R_{g}$ will be a time-dependent variable but will not change on the spherical cap. Likewise, the Schwarzschild parabola remains unchanged according to Birkhoff's theorem. It governs the course of the collapse by its shape. Therefore the quantity

$$
F_{1^{\prime}}=0, \quad F_{4^{\prime}}=\frac{1}{R_{g}} R_{g \mid 4^{\prime}}
$$

enters into the Einstein field equations. Primed indices indicate the comoving frame of reference. If we further make an ansatz for the collapse velocity, we are able to set up a Lorentz transformation which establishes the connection between comoving and non-comoving systems.

Since at any time of the collapse the state of the star is a snapshot of the interior Schwarzschild solution, which can be described by the structures explained in Section 2, we can always have access to the relations of this solution.

We note a relation known from the static model for our toolbox and complement it by an analogous one for the comoving system:

$$
\frac{1}{r} r_{11}=\frac{a_{R}}{r}, \quad r_{\mid 4}=0, \quad \frac{1}{r^{\prime}} r_{\mid 1^{\prime}}^{\prime}=\frac{a_{I}}{r}, \quad r_{\mid 4^{\prime}}^{\prime}=0 .
$$

The auxiliary variable $r^{\prime}$ has the range of values $\left[0, \mathrm{~L}, r_{g}^{\prime}\right] . r_{g}^{\prime}$ is the value of $r^{\prime}$ at the surface of the star. $r^{\prime}$ is referred to in the literature as comoving radial coordinate. But we do not make use of this interpretation, because we do not use or we are not able to use a coordinate system for the collapsing model. For this we have given reasons in detail above.

In our toolbox we place the following relations:

$$
\begin{aligned}
& a_{R}^{2}=1-\frac{r^{2}}{R_{g}^{2}}, \quad v_{R}=-\frac{r}{R_{g}}, \quad R_{g}=\sqrt{\frac{r_{g}^{3}}{2 M}} . \\
& a_{I}^{2}=1-\frac{r^{\prime 2}}{R_{0}^{2}}, \quad v_{I}=-\frac{r^{\prime}}{R_{0}}, \quad R_{0}=\sqrt{\frac{r_{g}^{\prime 3}}{2 M}}
\end{aligned}
$$

At the beginning of the collapse we have $r_{g}=r_{g}^{\prime}$ and $R_{g}=R_{0}$. We also demand that the two velocities $v_{R}, v_{I}$ defined in (3.3) are composed to the collapse velocity according to Einstein's addition law of velocities

$$
v_{C}=\frac{v_{R}-v_{I}}{1-v_{R} v_{I}} .
$$

With this we have set up the collapsing Schwarzschild model. The rest is tedious handwork.

The ansatz (3.3), (3.4) should be compared with historical papers. For $v_{I}=0$ one obtains the model of Oppenheimer and Snyder. For the surface of the OS-star one has $v_{R}^{g}=-r_{g} / R_{g}=-\sqrt{2 M / r_{g}}$. The pressure-free OS-star collapses in free fall from infinity. A combination of the two velocities other than (3.4), which violates the Einstein addition law of velocities, leads to the models of McVittie and Weinberg.

With (3.4) we are able to set up a Lorentz transformation and the accompanying Lorentz relations. We need a Lorentz transformation which connects a system which participates in the collapse and another which remains at rest relative to an observer system in the outer region

$$
\begin{aligned}
& L_{1}^{1}=\alpha_{C}, \quad L_{1}^{4}=i \alpha_{C} v_{C}, \quad L_{4}^{1}=-i \alpha_{C} v_{C}, \quad L_{4}^{4}=\alpha_{C} \\
& v_{C}=\frac{v_{R}-v_{I}}{1-v_{R} v_{I}}, \quad v_{R}=\frac{v_{C}+v_{I}}{1+v_{C} v_{I}}, \quad v_{I}=\frac{v_{R}-v_{C}}{1-v_{R} v_{C}} \\
& \alpha_{C}=\alpha_{R} \alpha_{I}\left(1-v_{R} v_{I}\right), \quad \alpha_{R}=\alpha_{C} \alpha_{I}\left(1+v_{C} v_{I}\right), \quad \alpha_{I}=\alpha_{C} \alpha_{R}\left(1-v_{C} v_{R}\right) \\
& \alpha_{C} v_{C}=\alpha_{R} \alpha_{I}\left(v_{R}-v_{I}\right), \quad \alpha_{R} v_{R}=\alpha_{C} \alpha_{I}\left(v_{C}+v_{I}\right), \quad \alpha_{I} v_{I}=\alpha_{R} \alpha_{C}\left(v_{R}-v_{C}\right)
\end{aligned}
$$


With the help of such a Lorentz transformation we can calculate the field quantities in the comoving system. Those field quantities, as set forth above, are components of the Ricci-rotation coefficients. Therefore we have to start from the inhomogeneous transformation law of the Ricci-rotation coefficients

$$
' A_{m^{\prime} n^{\prime}}^{s^{\prime}}=L_{m^{\prime} n^{\prime} s}^{m n s^{\prime}} A_{m n}^{s}+L_{s}^{s^{\prime}} L_{n^{\prime} \mid m^{\prime}}^{s} .
$$

The Ricci-rotation coefficients themselves are tensors. They describe the curvatures of the normal and oblique cuts through that surface, which we take as a basis of the theory. By the transformation

$$
A_{m n^{\prime}} s^{s^{\prime}}=L_{m n^{\prime} s^{\prime}}^{m n s^{\prime}} A_{m n}{ }^{s}
$$

the curvatures of the surface are not altered, but only adjusted to the point of view of a new observer. The above inhomogeneous transformation law has the meaning that a new object ' $A$ is assigned to the geometric object $A$. We call the second term in (3.6) Lorentz term and write it in short as

$$
L_{m^{\prime} n^{\prime}}^{s^{\prime}}=L_{s}^{s^{\prime}} L_{n^{\prime} \mid m^{\prime}}^{s}
$$

Thus, we end up in

$$
A_{m n^{\prime}}^{s^{\prime}}=A_{m^{\prime} n^{\prime}}^{s^{\prime}}+L_{m^{\prime} n^{\prime}}^{s^{\prime}} .
$$

However, concerning the collapse, the process will be somewhat more complex: we go back to the seed metric, we calculate the Lorentz term, we transform to a comoving system, we project to Schwarzschild, and we switch on the collapse by giving up the constraint $R_{g}=$ const. . Taken together in a formula this gives

$$
A_{m^{\prime} n^{\prime}}^{s^{\prime}}=P_{m^{\prime}}^{r^{\prime}}\left[L_{r^{\prime} n^{\prime} s}^{m s^{\prime}} \hat{A}_{m n}^{s}+\hat{L}_{r^{\prime} n^{\prime}}^{s^{\prime}}\right]=P_{m^{\prime}}^{r^{\prime}} A_{r^{\prime} n^{\prime}}{ }^{s}+L_{m^{\prime} n^{\prime}}^{s^{\prime}}, \quad L_{m^{\prime} n^{\prime}}^{s^{\prime}}=P_{m^{\prime}}^{r^{\prime}} L_{r^{\prime} n^{\prime}} s^{\prime} .
$$

We now expect the field quantities to have a fourth, time-like component in this system. It is easy to see that the lateral field quantities contained in the Ricci-rotation coefficients transform homogeneously

$$
\begin{aligned}
& B_{m^{\prime}}=L_{m^{\prime}}^{m} B_{m}=\left\{\alpha_{C} \frac{a_{R}}{r}, 0,0,-i \alpha_{C} v_{C} \frac{a_{R}}{r}\right\}, \\
& C_{m^{\prime}}=L_{m^{\prime}}^{m} C_{m}=\left\{\alpha_{C} \frac{a_{R}}{r}, \frac{1}{r} \cot \vartheta, 0,-i \alpha_{C} v_{C} \frac{a_{R}}{r}\right\},
\end{aligned}
$$

but the quantities $U$ transform inhomogeneously. We put the $U$-parts of the system into the form

$$
\hat{U}_{m^{\prime} n^{\prime}}^{s^{\prime}}=h_{m^{\prime}}^{s^{\prime}} U_{n^{\prime}}-h_{m^{\prime} n^{\prime}} U^{s^{\prime}}, \quad \text { 'U } U_{m^{\prime} n^{\prime}}^{s^{\prime}}=h_{m^{\prime}}^{s^{\prime}} \cdot U_{n^{\prime}}-h_{m^{\prime} n^{\prime}} U^{s^{\prime}} .
$$

First we get with [15 \# 13]

$$
\hat{U}_{m^{\prime}}=\left\{\alpha_{C}, 0,0,-i \alpha_{C} v_{C}\right\} \alpha_{R} v_{R} \frac{1}{R_{g}}
$$

and after the $P$-operation

$$
\begin{gathered}
U_{1^{\prime}}=P_{4^{\prime}}^{4^{\prime}} \hat{A_{4^{\prime} 1^{\prime}}}{ }^{4^{\prime}}, \quad U_{4^{\prime}}=P_{1^{\prime}}^{1^{\prime}} A_{1^{\prime} 4^{\prime}}{ }^{1^{\prime}}, \\
U_{m^{\prime}}=\left\{P \hat{U_{1^{\prime}}}, 0,0, U_{4^{\prime}}\right\} .
\end{gathered}
$$

At this stage of calculations the primed system is a moving system from which the static Schwarzschild metric is observed and is not connected to the collapse. Now the Lorentz term is to be calculated. From [15 \# 13] we take

and we write

$$
\hat{L}_{1^{\prime}}^{m a}=L_{4^{\prime} 1^{\prime}}^{4^{\prime}}=L_{s}^{4^{\prime}} L_{1^{\prime \prime} 4^{\prime}}^{s}=i \alpha_{C}^{2} v_{C \mid 4^{\prime}} \quad L_{4^{\prime}}={ }^{\prime} L_{1^{\prime} 4^{\prime}}{ }^{1^{\prime}}=-i \alpha_{C}^{2} v_{C \mid 1^{\prime}}
$$

$$
\hat{L}_{m^{\prime} n^{\prime}}^{{ }^{n}} s^{\prime}=h_{m^{\prime}}{ }^{s^{\prime}} L_{n^{\prime}}-h_{m^{\prime} n^{\prime}} L^{s^{\prime}} \text {. }
$$

The terms ' $\hat{L}$ are calculated [15 \# 14] and the P-operation is executed. With the auxiliary variables 


$$
\begin{aligned}
& G_{m^{\prime}}=\left\{i \alpha_{C} v_{C}, 0,0, \alpha_{C}\right\}\left(-i \alpha_{R} \frac{1}{R_{g}}\right), \quad l_{m^{\prime}}=\{0,0,0,1\} i \alpha_{I} v_{I} \frac{1}{r} \\
& f_{m^{\prime}}=\{1,0,0,0\}(1-P) \alpha_{C} v_{C} \alpha_{R} \frac{1}{R_{g}}, \quad g_{m^{\prime}}=\left\{-i \alpha_{I} v_{I}, 0,0, \alpha_{I}\right\} \frac{i}{R_{g}}=\hat{U}_{m^{\prime}}-G_{m^{\prime}}
\end{aligned}
$$

we finally obtain

$$
' L_{m^{\prime} n^{\prime}}^{s^{\prime}}=h_{m^{\prime}}^{s^{\prime}} L_{n^{\prime}}-h_{m^{\prime} n^{\prime}} L^{s^{\prime}}, \quad L_{m^{\prime}}=-\left[G_{m^{\prime}}-l_{m^{\prime}}-f_{m^{\prime}}\right],
$$

wherein the quantity $f$ is created by the $P$-operation. This reduces the inhomogeneous transformation law of the $U$-quantities to a vector equation [15 \# 15]

$$
\text { 'U } U_{m^{\prime}}=U_{m^{\prime}}+L_{m^{\prime}}, \quad \text { ' } U_{m^{\prime}}=\left\{P \alpha_{I} v_{I} \frac{1}{R_{g}}, 0,0,-i \alpha_{C} v_{C} a_{R} \frac{1}{r}\right\},
$$

in which the primes ahead of the kernel indicate a variable of the collapsing system. For the derivation of (3.17) we have used the Lorentz relations (3.5). We have implemented the collapse by considering the field quantities to be a function of time and by demanding that the primed reference system is connected to the collapse.

This is where the problem outlined in item 8) and 9) of the Introduction should be illuminated. If the quantity $U_{m^{\prime}}$ can be derived from the metric coefficients of a line element it can be represented as the gradient of a potential. Thus, ' $U_{m}$ ' can only be a gradient, if ' $L_{m}$ ' can also be brought into gradient form. However, this is in general not the case and is not the case in the present model either. The quantities cannot be based on a 4-bein system in such a way that its vectors are tangent to a comoving coordinate system. This in turn, makes the existence of a comoving coordinate system unlikely.

After looking at (3.10) and (3.17) reveals that one has

$$
\text { ' } U_{4^{\prime}} \stackrel{*}{=} B_{4^{\prime}} \stackrel{*}{=} C_{4^{\prime}} .
$$

From

$$
' u^{m^{\prime}} \| m^{\prime}=A_{m^{\prime}} u^{m^{\prime}}={ }^{\prime} U_{4^{\prime}}+B_{4^{\prime}}+C_{4^{\prime}}, \quad u^{m^{\prime}}=\{0,0,0,1\}
$$

we conclude that the contraction of a volume element is equal in all three directions. It still lacks the quantity mentioned in (3.1). It can be deduced from the field equations. However, we use the conservation law. The stressenergy tensor and its components can be taken over unchanged from the static model (2.25), provided that the indices will be primed. With [15 \# 16] one gains

$$
\mu_{04^{\prime}}=-\left(p+\mu_{0}\right)\left({ }^{\prime} U_{4^{\prime}}+B_{4^{\prime}}+C_{4^{\prime}}\right)^{\stackrel{*}{=}}-3\left(p+\mu_{0}\right)^{\prime} U_{4^{\prime}} .
$$

On the other hand (2.26), second equation, gives

$$
\mu_{0 \mid 4^{\prime}}=-2 \mu_{0} F_{4^{\prime}}
$$

and with the last two relations (2.26)

$$
F_{4^{\prime}}=(1-P)^{\prime} U_{4^{\prime}}=-i \alpha_{C} v_{C}(1-P) \frac{a_{R}}{r} .
$$

The conservation law of the comoving system [15 \# 17] is completely treated with

$$
T^{m^{\prime} n^{\prime} n^{\prime}}=0, \quad p_{\mid 1^{\prime}}=-\left(p+\mu_{0}\right)^{\prime} U_{1^{\prime}}, \quad \mu_{0 \mid 4^{\prime}} \stackrel{*}{=}-3\left(p+\mu_{0}\right)^{\prime} U_{4^{\prime}} .
$$

The quantity $F_{4}$, just derived enters into numerous calculations. The interested reader should follow the calculations [15 \# 18] which allow a more direct derivation of the quantities ' $L$. Since we did not obtain the field quantities by integration of the field equations, but by extending the static solution with the help of our toolbox, we now have to examine whether the field equations are satisfied with these field quantities. If we derive some auxiliary relations [15 \# 18] we finally obtain by use of the graded derivatives [15 \# 23, \# 24] 


$$
\begin{aligned}
& B_{m^{\prime} \| n^{\prime}}=B_{m^{\prime} \mid n^{\prime}}-U_{n^{\prime} m^{\prime}}{ }^{s^{\prime}} B_{s^{\prime}}, \quad C_{m^{\prime} \| n^{\prime}}=C_{m^{\prime} \mid n^{\prime}}-U_{n^{\prime} m^{\prime}}{ }^{s^{\prime}} C_{s^{\prime}}-B_{n^{\prime} m^{\prime}}{ }^{{ }^{\prime}} C_{s^{\prime}}
\end{aligned}
$$

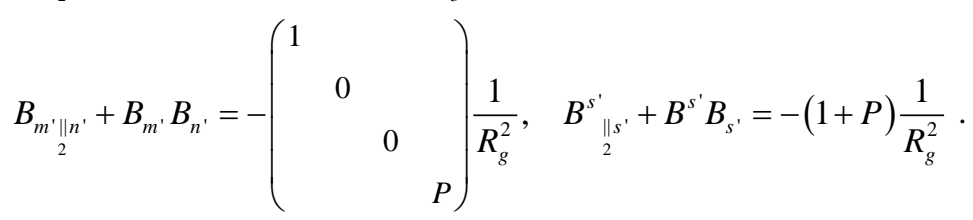

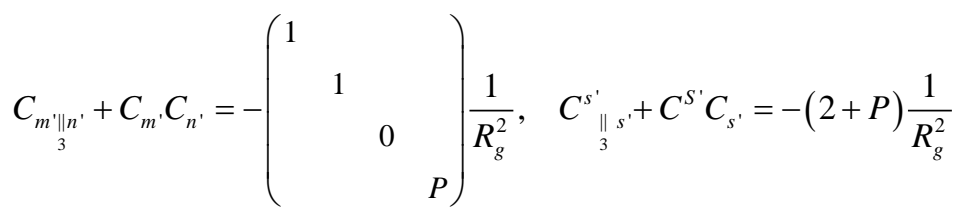

The verification of the ' $U$-equation is much more complex. We take from (3.17) the first relation at hand and we differentiate [15 \# 20 - \# 22]. It turns out that the $U$-part of the model is form-invariant

$$
' U^{s^{\prime}}{ }_{\mid s^{\prime}}+{ }^{\prime} U^{s^{\prime}} \cdot U_{s^{\prime}}=-\frac{P}{R_{g}^{2}} .
$$

For the calculation of this expression we have made use of the relation $P_{\mid 1^{\prime}}=(1-P)^{\prime} U_{1^{\prime}}$. Since there is no primed coordinate system, the relation cannot be obtained by direct differentiation, but must be recalculated via the static system. This gives the directly verifiable relation (2.29).

We can take over the structure of the field equations from (2.12), if we remove the hats and equip the kernels and indices with primes. If we substitute the calculated expressions of the subequations [15 \# 25, \# 26], the field equations are satisfied.

\section{Non-Comoving Observer System}

The model is only reasonable, if one succeeds in representing the field quantities in a non-comoving system and in resolving the field equations herewith. Having recalculated some auxiliary variables [15 \# 18], we also find the Lorentz term

$$
\begin{aligned}
& L_{m n}{ }^{s}=L_{s^{\prime}}^{s} L_{n \mid m}^{s^{\prime}}, \quad L_{m}=G_{m}-l_{m}-f_{m}, \quad L_{m}=-L_{m}^{m^{\prime}} L_{m^{\prime}} \\
& G_{m}=\{0,0,0,1\}\left(-i \alpha_{R} \frac{1}{R_{g}}\right), \quad l_{m}=\left\{-i \alpha_{C} v_{C}, 0,0, \alpha_{C}\right\} i \alpha_{I} v_{I} \frac{1}{r} \\
& f_{m}=\left\{\alpha_{C}, 0,0, i \alpha_{C} v_{C}\right\}(1-P) \alpha_{C} v_{C} \alpha_{R} \frac{1}{R_{g}}, \quad g_{m}=\left\{-i \alpha_{R} v_{R}, 0,0, \alpha_{R}\right\} \frac{i}{R_{g}}=\hat{U}_{m}-G_{m}
\end{aligned}
$$

With this we can calculate the inhomogeneously transforming $U$-quantities [15 \# 27]. We realize that the noncomoving system is not identical with the static system, because we have not dismissed the condition $R_{g} \neq$ const. . We perform the following operations

$$
B_{m}=L_{m}^{m^{\prime}} B_{m^{\prime}}, \quad C_{m}=L_{m}^{m^{\prime}} C_{m^{\prime}}, \quad U_{m}=L_{m}^{m^{\prime}} \cdot U_{m^{\prime}}+L_{m} .
$$

The lateral quantities take the static form. However, for the quantity $U$

$$
U_{m}=P \hat{U}_{m}-\alpha_{R}^{2} v_{R}^{2} F_{m}=-E_{m}-\alpha_{R}^{2} v_{R}^{2} F_{m}, \quad F_{m}=L_{m}^{m^{\prime}} F_{m^{\prime}}
$$

applies.

The first term in (4.3) is the Schwarzschild gravity inside the star, the second the acceleration of the particles in the interior which is caused by the collapse. It can be seen that the expression only goes over into the static one, if we switch off the collapse, thus if we put $R_{g}=$ const., $\left(F_{m}=0\right)$. The $U$-equation again is form-invariant [15 \# 28, \# 29]

$$
U^{s}{ }_{1}+U^{s} U_{s}=-\frac{P}{R_{g}^{2}} .
$$


In contrast, for the $B$ - and $C$-equations we get with [15 \# 30]

$$
\begin{aligned}
& \underset{\substack{m \| n \\
2}}{B_{n \mid n}}=B_{m \mid n}-U_{n m}^{s} B_{s}, \quad C_{\substack{m \| n \\
3}}=C_{m \mid n}-U_{n m}^{s} C_{s}-B_{n m}{ }^{s} C_{s}
\end{aligned}
$$

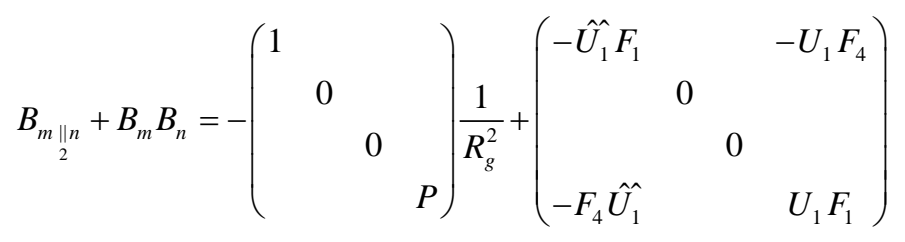

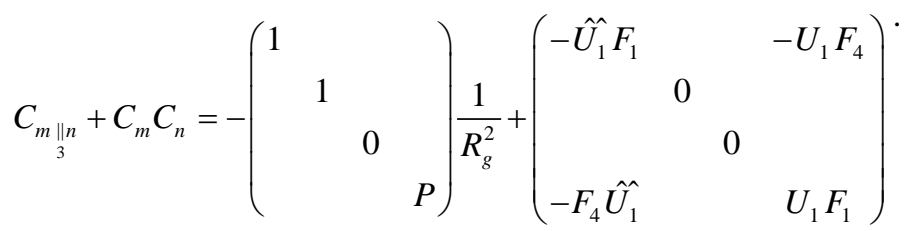

$$
\begin{aligned}
& B_{\substack{\| s \\
s}}+B^{s} B_{s}=-(1+P) \frac{1}{R_{g}^{2}}, \quad C_{\substack{\| s \\
s}}^{s}+C^{s} C_{s}=-(2+P) \frac{1}{R_{g}^{2}}
\end{aligned}
$$

This allows us to represent the Einstein tensor completely. We only have to prepare the right side of the field equations. The stress-energy tensor in the non-comoving system

$$
T_{m n}=-p g_{m n}+\left(p+\mu_{0}\right)^{\prime} u_{m}{ }^{\prime} u_{n}, \quad ' u_{m}=\left\{-i \alpha_{C} v_{C}, 0,0, \alpha_{C}\right\}
$$

we write component by component

$$
\begin{aligned}
& T_{11}=-p-\alpha_{C}^{2} v_{C}^{2}\left(p+\mu_{0}\right), \quad T_{22}=-p, \quad T_{33}=-p, \\
& T_{41}=-i \alpha_{C}^{2} v_{C}\left(p+\mu_{0}\right), \quad T_{44}=\mu_{0}+\alpha_{C}^{2} v_{C}^{2}\left(p+\mu_{0}\right)
\end{aligned} .
$$

The question arises of whether the stress-energy tensor can be geometrized, i.e. whether the quantities of the right side of the field equations can be brought into connection with the very different field quantities of the left side. If we calculate the quantities in the second brackets of (4.5) [15 \# 31]

$$
2 \hat{U_{1}} F_{1}=\alpha_{C}^{2} v_{C}^{2} \kappa\left(p+\mu_{0}\right), \quad 2 U_{1} F_{4}=i \alpha_{C}^{2} v_{C} \kappa\left(p+\mu_{0}\right)
$$

it is evident that the lateral subequations establish the necessary connection. If one also takes into consideration the $U$-equation, one has geometrized the stress-energy tensor.

\section{Discussion of the Model}

In the preceding sections it has been suggested that a star which is subjected to the Schwarzschild collapse can contract only up to a minimum radius $r_{h}$. We will show that this radius can be reached only asymptotically. First, we derive from

$$
r_{\mid 4^{\prime}}=L_{4}^{1} r_{11}=-i \alpha_{C} v_{C} a_{R}, \frac{\alpha_{R} \mathrm{~d} r}{i \mathrm{~d} T^{\prime}}=-i \alpha_{C} v_{C}
$$

the collapse velocity. In the system in rest one has $\mathrm{d} x^{1}=\alpha_{R} \mathrm{~d} r$, in the commoving $\mathrm{d} x^{1^{\prime}}=0$. For the connection of the proper times the Lorentz relation $\mathrm{d} T / \mathrm{d} T^{\prime}=\alpha_{C}$ applies. With this one obtains from the above expression the collapse velocity

$$
v_{C}=\frac{\mathrm{d} x^{1}}{\mathrm{~d} T},
$$

which we refer to the surface of the star. At this location is $r=r_{g}, r_{g}^{\prime}=r_{0}=$ const. From

$$
\frac{\alpha_{R} \mathrm{~d} r}{\mathrm{~d} T^{\prime}}=\alpha_{C} v_{C}=\alpha_{R} \alpha_{I}\left(v_{R}-v_{I}\right)
$$

we obtain 


$$
\mathrm{d} r=\alpha_{I}\left(v_{R}-v_{I}\right) \mathrm{d} T^{\prime}
$$

or taking into account (3.3)

$$
\mathrm{d} T^{\prime}=\frac{1}{\alpha_{I} v_{I}} \frac{\sqrt{r}}{\sqrt{r_{0}}-\sqrt{r}} \mathrm{~d} r .
$$

In it are $\alpha_{I}, v_{I}$, and $r_{0}$ constants. Integration results in a function

$$
f(r)=-\frac{1}{\alpha_{I} v_{I}}\left[r+2 \sqrt{r_{0}} \sqrt{r}+2 r_{0} \ln \left(\sqrt{r_{0}}-\sqrt{r}\right)\right],
$$

which is to be regarded in the range $\left[r_{h}, r_{0}\right]$. Since $r$ is an outgoing coordinate the collapse, however, is directed inwards, we shift the origin of the coordinate system to the position of the surface, and that at the beginning of the collapse. We let run inwards the new radial coordinate $r \rightarrow r_{0}-r, r_{h} \rightarrow r_{0}-r_{h}$. Then we have

$$
g(r)=r_{0}-r+2 \sqrt{r_{0}-r_{h}} \sqrt{r_{0}-r}+2\left(r_{0}-r_{h}\right) \ln \left(\sqrt{r_{0}-r_{h}}-\sqrt{r_{0}-r}\right) .
$$

If we choose the constant of integration as $g\left(r_{0}\right)=2\left(r_{0}-r_{h}\right) \ln \sqrt{r_{0}-r_{h}}$, then the proper time at the beginning of the collapse is $T^{\prime}=0$. Finally, one has in the range under consideration

$$
T^{\prime}(r)=-\frac{1}{\alpha_{I} v_{I}}\left[g(r)-g\left(r_{0}\right)\right] .
$$

The function is depicted in Figure 2.

From this figure, one can gather how much time has passed, if the surface of the star has moved a certain distance $r_{0}-r$. From $\lim _{r \rightarrow r_{h}} T^{\prime}(r)=\infty$ is apparent that the star needs an infinitely long time to reach the minimum radius. Thus, the collapsing interior Schwarzschild solution has an inner horizon. It is identical to the abovementioned pressure horizon and the velocity horizon. The star can never shrink to a point. The matter density, the pressure, and the curvature of space are never infinite. The inner horizon is above the event horizon of the exterior Schwarzschild solution.

\section{Conclusion}

The formation of a black hole in this model is not possible. Our model describes an ECO (eternally collapsing object), as was predicted by Mitra [16] on the basis of astrophysical considerations. Since the exterior Schwarzschild solution has been proven and describes Nature well, one can assume that the interior solution can describe the interior of a star at least in a rough approximation. Although the two parameters, pressure and mass density, are not sufficient to record the properties of a star, there is still hope that at least some basic properties of the

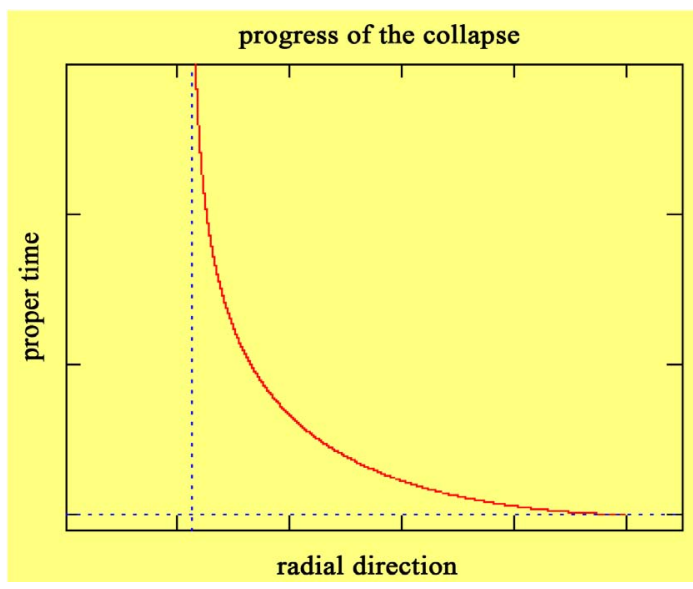

Figure 2. The star needs an infinitely long time to reach the minimum radius. 
model have general validity and that also more pretentious models do not exhibit unusual behaviors.

\section{References}

[1] Oppenheimer, J.R. and Snyder, H. (1939) On Continued Gravitational Contraction. Physical Review, 56, 455-459. http://dx.doi.org/10.1103/PhysRev.56.455

[2] Burghardt, R. (2014) Spacetime Curvature. 1-480. http://www.arg.or.at/EMono.htm

[3] Israel, W. (1958) Discontinuities in Spherically Symmetric Gravitational Fields and Shells of Radiation. Proceedings of the Royal Society of London A, 248, 404-414. http://dx.doi.org/10.1098/rspa.1958.0252

[4] O’Brien, S. and Synge, J.L. (1952) Jump Conditions at Discontinuities in General Relativity. Communications of the Dublin Institute for Advanced Studies, No. 9, 1-20.

[5] Robson, E.H. (1972) Junction Condition in General Relativity Theory. Annales de l'Institut Henri Poincaré, 16, 41-50.

[6] Bonnor, W.B. and Vickers, P.A. (1981) Junction Conditions in General Relativity. General Relativity and Gravitation, 13, 29-36.

[7] Nariai, H. (1965) On the Boundary Conditions in General Relativity. Progress of Theoretical Physics, 34, $173-186$. http://dx.doi.org/10.1143/PTP.34.173

[8] Lichnerowicz, A. (1955) Théories relativistes de la gravitation et de l'électromagnétisme. Masson et $C^{\text {ie }}$, Paris.

[9] Nariai, H. and Tomita, K. (1966) On the Applicability of a Dust-Like Model to a Collapsing or Anti-Collapsing Star at High Temperature. Progress of Theoretical Physics, 35, 777-785. http://dx.doi.org/10.1143/PTP.35.777

[10] Nariai, H. and Tomita, K. (1965) On the Problem of Gravitational Collapse. Progress of Theoretical Physics, 34, 155172. http://dx.doi.org/10.1143/PTP.34.155

[11] McVittie, G.C. (1967) Gravitational Motions of Collapse or of Expansion in General Relativity. Annales de l'Institut Henri Poincaré, 6, 1-15.

[12] McVittie, G.C. (1964) Gravitational Collapse to a Small Volume. Astrophysical Journal, 140, 401-416. http://dx.doi.org/10.1086/147937

[13] Weinberg, S. (1997) Gravitation and Cosmology. John Wiley \& Sons, New York.

[14] Burghardt, R. (2009) Interior Schwarzschild Solution and Free Fall. Report ARG-2009-06, 1-8. http://arg.or.at/

[15] Burghardt, R. (2015) Hilfedatei für “Collapsing Schwarzschild interior”. 1-15. http://www.arg.or.at/PendingPapers/HelpFile.pdf

[16] Mitra, A. (2006) Black Holes or Eternally Collapsing Objects: A Revue of 90 Years of Misconception. Focus of Black Hole Research. Nova Science Publishers, New York, 1-97. 